\title{
CAMBIOS EN LA PERCEPCIÓN DEL PROFESORADO SOBRE LA INCIDENCIA POSITIVA DE LAS TECNOLOGÍAS DIGITALES EN EDUCACIÓN PRIMARIA Y SECUNDARIA
}

\author{
Juan Bosco López González \\ Dallas Independent School District \\ Juana María Ortega Tuleda \\ Universidad de Jaén
}

\begin{abstract}
Resumen. En este estudio se describe y relaciona la evolución producida en la opinión del profesorado de Primaria y Secundaria sobre la incidencia positiva de las TIC en el proceso educativo. Se presentan los resultados alcanzados con la aplicación de un cuestionario $(n=744)$. La investigación no experimental se llevó a cabo mediante un estudio longitudinal de tendencia en 2009 y 2016. De esta manera, con dos muestras distintas de una misma población, se pudo relacionar y contrastar la evolución de la repercusión positiva de las TIC en la práctica educativa. Entre los resultados se encontraron cuatro variables que se relacionaron con la opinión del profesorado sobre la incidencia positiva de las TIC en la educación. Estas variables fueron: el uso de las tecnologías digitales en el aula, el nivel de formación del profesorado en tecnologías digitales, la consideración que el docente tiene para integrar las TIC en la práctica docente, y la etapa educativa donde el profesorado imparte docencia.

Palabras clave: Educación primaria, educación secundaria, TIC, impacto, percepción.
\end{abstract}

\section{CHANGES IN TEACHERS' PERCEPTION OF THE POSITIVE IMPACT OF DIGITAL TECHNOLOGY ON PRIMARY AND SECONDARY EDUCATION.}

\begin{abstract}
This study describes and relates the evolution produced in the opinion of Primary and Secondary teachers about the positive impact of information and communication technology (ICT) in the educational process. Results are presented with the application of a questionnaire $(n=744)$. Nonexperimental research was carried out through a longitudinal trend study in 2009 and 2016. Thus, with two different samples from the same population, it was possible to relate and contrast the evolution of the positive impact of ICT in educational practice. Among the results were four variables that were related to the opinion of teachers on the positive incidence of digital technology in education. These variables were: the use of digital technology in the classroom, the level of teacher training in digital technology, the consideration that the teacher has to integrate ICT in teaching practice, and the educational stage where teachers teach.
\end{abstract}

Keywords: Primary education, secondary education, ICT, impact, perception. 


\title{
MUDANÇAS NA PERCEPÇÃO DOS PROFESSORES SOBRE A INCIDÊNCIA POSITIVA DAS TECNOLOGIAS DIGITAIS NO ENSINO PRIMÁRIO E SECUNDÁRIO
}

\begin{abstract}
Resumo. Neste estudo descreve-se e relaciona-se a evolução produzida, na opinião dos professores de ensino primário e secundário, sobre a incidência das TIC no processo educativo. Os resultados atingidos são apresentados com a aplicação de um questionário $(n=744)$. A investigação não experimental foi feita através de um estudo longitudinal de tendência em 2009 e 2016. Assim, com duas amostras diferentes de uma mesma população, pôde-se relacionar e contrastar a evolução da repercussão positiva das TIC na prática educativa. Entre os resultados, encontraram-se quatro variáveis que foram relacionadas com a opinião do conjunto dos professores sobre a incidência positiva das TIC na Educação. Estas variáveis foram: o uso das tecnologias digitais nas salas das aulas, o nível de formação dos professores em tecnologias digitais, a consideração que os professores têm para integrar as TIC na prática docente, e a etapa educativa em que o professor ensina.
\end{abstract}

Palavras-chave: Educação primária, educação secundária, TIC, impacto, percepção.

\section{Introducción}

Las tecnologías digitales avanzan rápidamente, si bien los cambios en los procesos educativos se introducen con lentitud. Los centros educativos, paso a paso, han ido incorporando más recursos tecnológicos y nuevas metodologías en la tarea docente, aunque no siempre han respondido a las finalidades de un plan, ni sus avances han sido evaluados convenientemente para guiar sucesivos logros.

Hay gran diversidad de estudios que describen pormenorizadamente cómo ha sido el proceso de incorporación de las TIC en los centros educativos (Cebrián y Ruiz, 2008; Cebrián, Ruiz y Sánchez, 2008; Pérez, Aguaded y Fandos, 2009; Colás, De Pablos, González y Conde, 2009; Ruiz y Ortega, 2009; Aguaded y Pérez, 2010; Area, 2010; Area, Sanabria y Vega, 2015).

Esta investigación se enmarca en lo que Area (2005) clasifica como estudios sobre las perspectivas, opiniones y actitudes de los agentes educativos hacia las Tecnologías de la Información y la Comunicación (TIC) y las prácticas de uso de ordenadores en los contextos escolares. Los estudios de Chiero, Windschitl y Sahl sostienen que "la necesidad de realización de estos estudios se apoya en el supuesto de que las prácticas de enseñanza con ordenadores está condicionada, entre otros factores, por lo que piensan los docentes en torno al potencial pedagógico de dichas tecnologías, por las actitudes que mantienen hacia las mismas y hacia la innovación educativa" (citado en Area, 2005).

En diversas ocasiones, al identificar las actitudes y opiniones del profesorado que usa las tecnologías digitales en su práctica docente no se distinguen cambios significativos en los procesos de enseñanza-aprendizaje, ni en las expectativas del cambio metodológico con las que parten los docentes (Cebrián, Ruiz y Sánchez, 2008). Nos encontramos, sin embargo, con una variedad de centros TIC que han superado muchas de estas dificultades, siendo referentes de buenas prácticas para otros centros, (Boza, Toscano y Méndez, 2009; Boza y Toscano, 2011; Cabero, 2010; Cebrián, Sánchez, Ruiz \& Palomo, 2011; De Pablos, Colás, \& Villaciervos, 2010; Area, Borrás, Castro, Cepeda, Fariña, González y Sanabria, 2013; Valverde y Sosa, 2014). No faltando ejemplos de prácticas en el uso de las tecnologías digitales para favorecer la 
inclusión del alumnado. "Hablar de las TIC para favorecer la inclusión educativa, es referirnos también a las posibilidades que nos ofrecen para mejorar las condiciones de vida de colectivos tradicionalmente marginados" (Cabero y Fernández, 2014, p10).

Paraskeva, Bouta y Papagianni (2008) afirman que las creencias que los docentes conservan sobre su propia eficacia docente están relacionadas con sus prácticas, en consecuencia las actitudes favorables hacia las tecnologías y una percepción positiva de la propia competencia digital se han mostrado como condiciones previas para la incorporación del ordenador en la enseñanza.

Viherä y Nurmela (2001) plantean que existen tres condiciones elementales para que un docente comience a utilizar TIC en el aula: El nivel de acceso o la disponibilidad de TIC en el centro docente, la competencia o nivel de dominio del propio profesor en el uso de las TIC y la actitud general hacia el uso de las tecnologías en el aula.

En numerosas investigaciones se destaca la importancia que tienen las concepciones, creencias y actitudes del profesorado en el proceso de incorporación de las tecnologías digitales a su práctica docente (Jimoyiannis y Komis, 2007; Hermans, Tondeur, Braak y Valcke, 2008; Sang, Valcke, Braak y Tondeur, 2010; Fu, 2013). Estos estudios analizan el impacto que tienen las opiniones del profesorado sobre la utilización que hacen de las TIC en el aula. Los resultados defienden una estrecha relación entre estas variables. También encontramos en el contexto andaluz antecedentes sobre el estudio de las concepciones y creencias que los docentes sostienen sobre el uso de las TIC. Boza, Tirado y Guzmán (2010) concluyen que los profesores que tienen alguna creencia formada sobre la integración de la tecnología en la educación “están más cualificados tecnológicamente que los incrédulos, se sienten más satisfechos con la formación en la que se implicaron, con los recursos, materiales e infraestructura tecnológica, asimismo es más normal en ellos que hagan un uso frecuente de las tecnologías y de aquellas aplicaciones más novedosas" (p.14).

Tirado y Aguaded (2014), en otra investigación sobre este tema, sostienen que las creencias del profesorado sobre el sentido y significado de las tecnologías en la educación actúan como facilitadores o barreras del uso regular de TIC en el aula.

Teniendo en cuenta estas consideraciones, el origen de esta investigación parte de las siguientes preguntas clave: ¿Hay relación entre la opinión que tiene el profesorado sobre las TIC y la utilización de éstas con su alumnado?¿Qué variables se relacionan con la incorporación de las tecnologías en los centros educativos? ¿La formación en TIC del profesorado y la consideración que tiene de su capacidad para integrar las TIC en el aula está ligada a procesos de cambio en su práctica docente?

Este estudio, en coherencia con los planteamientos teóricos, tuvo por objetivo contrastar y relacionar los cambios en la opinión del profesorado sobre la incidencia positiva de las TIC en el proceso educativo.

\section{Método}

\section{Población y muestra}

La población de este estudio la constituye el profesorado de Educación Primaria y Educación Secundaria de los centros participantes en un Proyecto Educativo de Centro para la incorporación de las Tecnologías de la Información y la Comunicación a la práctica docente, en los centros TIC de la provincia de Jaén.

Se realizaron dos muestreos en dos momentos diferentes. Uno en 2009 y otro en 2016. La muestra productora de 2009 fue de 372 docentes $(n=372)$, pertenecientes a 29 centros educativos, 17 de Educación Primaria y 12 de Secundaria. La edad media de los participantes fue de 42,03 años (DT = 9,83). El 54,6\% fueron docentes de Educación Primaria y el 45,4\% de Secundaria. El 50\% fueron mujeres y el 50\% hombres. 
Los sujetos que componían la primera muestra se obtuvieron a través de un muestreo no probabilístico, del tipo intencional u opinático. Este tipo de muestreo, en palabras de Buendía, Colás y Hernández, (1997) estudia "los elementos típicos o representativos de cierta población, ya que la forma de asegurarse de que se incluirán en dicha investigación, es elegirlos intencionalmente". Con este método se seleccionaron los centros TIC que tenían al menos dos años de experiencia en el proyecto. El profesorado se seleccionó posteriormente de entre estos centros.

Para mantener estables las características del contexto de la investigación, en la segunda muestra de 2016 se seleccionaron los mismos centros educativos que la primera. En consecuencia, esta segunda muestra fue también de 372 docentes $(n=372)$.

La muestra total fue de 744 docentes $(n=744)$. Entre ambas muestras no se hallaron diferencias significativas en cuanto al género, la edad del profesorado, ni a la etapa educativa en la que impartieron docencia.

\section{Metodología}

Teniendo en cuenta el problema de estudio y los objetivos, esta investigación es de tipo no experimental. La metodología llevada a cabo responde a un diseño longitudinal de tendencia. "Este tipo de estudios describen el cambio mediante la selección de muestras distintas para cada momento temporal de recogida de información" (Bisquerra, 2009, p.201). Con este diseño se pudo comparar y contrastar la evolución de la finalidad del uso de las TIC en la práctica docente, con dos muestras de la misma población en dos momentos diferentes.

\section{Instrumentos}

Se recopilaron los datos mediante el "Cuestionario sobre el desarrollo de Proyectos TIC para la incorporación de las Tecnologías de la Información y la Comunicación en los centros de Educación Primaria y Secundaria de Andalucía" (Ruiz y Ortega, 2009). El cuestionario contempla tres dimensiones en relación a la incorporación y uso de las TIC. Por su extensión, este artículo se centra en una parte de los resultados relativos a la "incidencia positiva de las TIC en el proceso educativo". Esta variable está formada por 18 ítems de una escala tipo Likert de respuesta de cuatro niveles. La fiabilidad del cuestionario a nivel global tiene un coeficiente alfa de Cronbach de 0,955. El valor de la dimensión "Incidencia positiva de las TIC" fue 0,931.

\section{Procedimiento}

En una primera fase se contactó con los directores de los centros escolares seleccionados. Se les informó de la investigación y se les invitó a la participación. En otra cita posterior, en cada centro, se informó a los docentes que voluntariamente quisieron participar en la investigación. La administración del cuestionario se llevó a cabo a través de dos medios. El primero, mediante la administración individual del cuestionario a los docentes que voluntariamente aceptaron participar. Este procedimiento supuso el $90 \%$ de los casos. El segundo, mediante la administración colectiva, en reuniones en los centros, suponiendo el otro $10 \%$ restante de casos. Estos dos medios también se utilizaron para administrar los cuestionarios en 2016.

\section{Resultados}

La finalidad de esta investigación fue conocer la evolución entre 2009 y 2016 de la percepción del profesorado sobre la incidencia positiva de las tecnologías digitales en la educación.

Los resultados mostraron que el profesorado tuvo una visión positiva de las tecnologías digitales, entre otros motivos, porque facilita el acceso a la información, suscita el interés y la motivación del alumnado, y promueve la participación de éste. Estas valoraciones fueron comunes entre el profesorado en 2009 y 2016. Además, desde 
2009 hasta 2016, la valoración que más aumentó entre el profesorado fue que las TIC son imprescindibles en la práctica educativa.

Al preguntar la valoración del profesorado sobre la incidencia positiva de las TIC en el proceso educativo se le plantearon dieciocho ítems: las TIC facilitan el acceso a la información (ítem 1), las TIC mejoran la adquisición de conocimientos por parte del alumnado (ítem 2), las TIC promueven la participación del alumnado (ítem 3), las TIC suscitan interés y motivación del alumnado (ítem 4), la utilización de las TIC facilita el acceso al currículo del alumnado con necesidades educativas especiales (N.E.E.) (ítem 5), el uso de las TIC facilita la atención a la diversidad (ítem 6), la incorporación de las TIC en la enseñanza mejora el rendimiento escolar del alumnado (ítem 7), las TIC favorecen dinámicas de trabajo en equipo en el alumnado (ítem 8), las TIC promueven un aprendizaje más autónomo del alumnado (ítem 9), las TIC posibilitan una enseñanza más personalizada (ítem 10), las TIC potencian la interacción profesorado-alumnado (ítem 11), la utilización de las TIC en la enseñanza exige un cambio metodológico importante (ítem 12), las TIC favorecen el trabajo colaborativo del profesorado (ítem 13), las TIC son imprescindibles en la práctica educativa actual (ítem 14), es necesaria su utilización para poder desenvolverse en la sociedad (ítem 15), el uso de las TIC favorece el uso de dinámicas de trabajo innovadoras y creativas (ítem 16), las TIC conllevan cambios positivos en el rol del profesorado y del alumnado (ítem 17), el uso de las TIC tiene una incidencia clara y positiva en el proceso de aprendizaje del alumnado (ítem 18).

Tabla 1

Estadísticos de la variable 'incidencia positiva de las TIC en el proceso educativo', 2009-2016

\begin{tabular}{|c|c|c|c|c|c|c|c|}
\hline Ítems & $\begin{array}{c}2009 \\
M(D T)\end{array}$ & $\begin{array}{c}2016 \\
M(D T)\end{array}$ & $t$ & $p$ & & & $\begin{array}{l}\mathrm{TE} \\
(r)\end{array}$ \\
\hline $\begin{array}{l}\text { Facilitan el acceso a la } \\
\text { información }\end{array}$ & $3,39(0,62)$ & $3,60(0,52)$ & 4,24 &, $00^{* *}$ & 0,11 & 0,31 & 0,18 \\
\hline $\begin{array}{l}\text { Mejoran la adquisición de } \\
\text { conocimientos }\end{array}$ & $3,04(0,63)$ & $3,29(0,62)$ & 4,69 &, $00^{* *}$ & 0,15 & 0,36 & 0,20 \\
\hline $\begin{array}{l}\text { Promueven la participación } \\
\text { del alumno }\end{array}$ & $3,17(0,61)$ & $3,29(0,64)$ & 2,25 &, $03^{*}$ & 0,02 & 0,23 & 0,10 \\
\hline $\begin{array}{l}\text { Suscitan interés y } \\
\text { motivación del alumnado }\end{array}$ & $3,33(0,63)$ & $3,43(0,57)$ & 1,86 & ,06 & $-0,01$ & 0,20 & 0,08 \\
\hline $\begin{array}{l}\text { Facilita el acceso al } \\
\text { currículo del alumnado con } \\
\text { NEE }\end{array}$ & $2,96(0,65)$ & $3,21(0,66)$ & 4,27 &, $00^{* *}$ & 0,13 & 0,36 & 0,18 \\
\hline $\begin{array}{l}\text { Facilita la atención a la } \\
\text { diversidad }\end{array}$ & $3,02(0,62)$ & $3,21(0,65)$ & 3,46 &, $00^{* *}$ & 0,08 & 0,30 & 0,15 \\
\hline $\begin{array}{l}\text { Mejora el rendimiento } \\
\text { escolar del alumnado }\end{array}$ & $2,70(0,70)$ & $2,96(0,71)$ & 4,26 &, $00^{* *}$ & 0,14 & 0,38 & 0,18 \\
\hline $\begin{array}{l}\text { Favorecen dinámicas de } \\
\text { trabajo en equipo }\end{array}$ & $2,87(0,68)$ & $3,08(0,70)$ & 3,46 &, $00^{* *}$ & 0,09 & 0,33 & 0,15 \\
\hline $\begin{array}{l}\text { Promueven un aprendizaje } \\
\text { más autónomo }\end{array}$ & $3,06(0,67)$ & $3,16(0,64)$ & 1,83 & ,07 & $-0,01$ & 0,22 & 0,08 \\
\hline $\begin{array}{l}\text { Posibilitan una enseñanza } \\
\text { más personalizada }\end{array}$ & $2,92(0,63)$ & $3,02(0,72)$ & 1,68 & ,10 & $-0,02$ & 0,22 & 0,07 \\
\hline $\begin{array}{l}\text { Potencian la interacción } \\
\text { profesorado-alumnado }\end{array}$ & $2,84(0,70)$ & $2,94(0,74)$ & 1,66 &, 10 & $-0,02$ & 0,23 & 0,07 \\
\hline $\begin{array}{l}\text { Exige un cambio } \\
\text { metodológico importante }\end{array}$ & $3,29(0,68)$ & $3,17(0,73)$ & $-1,85$ & ,07 & $-0,24$ & 0,01 & 0,08 \\
\hline $\begin{array}{l}\text { Favorecen el trabajo } \\
\text { colaborativo del profesorado }\end{array}$ & $3,01(0,66)$ & $3,00(0,71)$ & $-0,09$ & ,93 & $-0,12$ & 0,11 & 0,00 \\
\hline
\end{tabular}




\begin{tabular}{|c|c|c|c|c|c|c|c|}
\hline Ítems & $\begin{array}{c}2009 \\
M(D T)\end{array}$ & $\begin{array}{c}2016 \\
M(D T)\end{array}$ & $t$ & $p$ & $\begin{array}{l}\text { IC } \\
\text { Inf. }\end{array}$ & $\begin{array}{l}75 \% \\
\text { Sup. }\end{array}$ & $\begin{array}{l}\mathrm{TE} \\
(r)\end{array}$ \\
\hline $\begin{array}{l}\text { Son imprescindibles en la } \\
\text { práctica educativa actual }\end{array}$ & $2,80(0,78)$ & $3,16(0,74)$ & 5,46 &, $00^{* *}$ & 0,23 & 0,49 & 0,23 \\
\hline $\begin{array}{l}\text { Es necesaria su utilización } \\
\text { para poder desenvolverse en la } \\
\text { sociedad }\end{array}$ & $3,11(0,67)$ & $3,37(0,60)$ & 4,81 &, $00^{* *}$ & 0,16 & 0,37 & 0,20 \\
\hline $\begin{array}{l}\text { Favorece el uso de } \\
\text { dinámicas de trabajo } \\
\text { innovadoras y creativas }\end{array}$ & $3,14(0,54)$ & $3,23(0,62)$ & 1,73 & ,08 & $-0,01$ & 0,19 & 0,07 \\
\hline $\begin{array}{l}\text { Conllevan cambios } \\
\text { positivos en el rol del } \\
\text { profesorado y del alumno }\end{array}$ & $2,99(0,64)$ & $3,06(0,68)$ & 1,21 &, 23 & $-0,04$ & 0,18 & 0,05 \\
\hline $\begin{array}{l}\text { Tiene una incidencia clara } \\
\text { y positiva en el proceso de } \\
\text { aprendizaje }\end{array}$ & $3,00(0,62)$ & $3,20(0,63)$ & 3,68 &, $00^{* *}$ & 0,09 & 0,31 & 0,16 \\
\hline
\end{tabular}

En 2009, según los datos del estudio, los docentes valoraron que las TIC incidían positivamente en los procesos de enseñanza y aprendizaje, ya que facilitaban el acceso a la información $(M=3,39, D T=0,62)$, suscitaban el interés y la motivación del alumnado $(M=3,33, D T=0,63)$, y aunque exigía un cambio metodológico importante $(M=3,29, D T=0,68)$ promovían la participación del alumnado $(M=3,17, D T=0,61)$.

En 2016, el profesorado valoró positivamente que las TIC facilitaban el acceso a la información $(M=3,60, D T=0,52)$ y seguían suscitando el interés y motivación del alumnado $(M=3,43, D T=0,57)$. Con el paso del tiempo, como novedad con respecto al primer año, los docentes valoraron que las TIC eran necesarias para desenvolverse en la sociedad $(M=3,37, D T=0,60)$, mejoraban la adquisición de conocimientos $(M=3,29$, $D T=0,62)$ y promovían la participación del alumnado $(M=3,29, D T=0,64)$.

Entre 2009 y 2016 hubo aspectos que se mantuvieron estables en la opinión del profesorado y otros, sin embargo, se vieron modificados. Se hallaron cambios significativos en la opinión del profesorado en cuanto a la incidencia de las TIC.

Uno de los cambios en la opinión del profesorado es que las TIC comienzan a ser imprescindibles en la práctica educativa en una cantidad significativa de docentes [ $\mathrm{t}$ $(742)=5,46 ; \mathrm{p}=, 00,(r=0,23)]$. Con menor incremento desde 2009, también se valoró positivamente las TIC por ser necesarias para poder desenvolverse en la sociedad [t $(742)=4,81 ; \mathrm{p}=, 00 ; r=0,20]$; porque mejoran la adquisición de los conocimientos [t $(742)=4,69 ; \mathrm{p}=, 00 ; r=0,20]$; porque facilitan el acceso a la información [t $(742)=$ $4,24 ; \mathrm{p}=, 00 ; r=0,18]$, porque facilitan el acceso al currículo del alumnado con NEE [t $(742)=4,27 ; \mathrm{p}=, 00 ; r=0,18]$ y porque tiene una incidencia clara y positiva en el proceso de aprendizaje [ $\mathrm{t}(742)=3,68 ; \mathrm{p}=, 00 ; r=0,16]$.

Diferencias entre el profesorado sobre la incidencia positiva de las TIC en el proceso educativo.

Se encontraron tres variables relacionadas con los docentes que mostraron una opinión más positiva sobre la incidencia de las TIC en la educación.

En primer lugar, se observó que había diferencias en las percepciones del profesorado que consideraba tener un nivel suficiente para integrar las TIC en la práctica docente. En segundo lugar, el profesorado con un nivel medio o avanzado en TIC también difirió de quienes tenían un nivel básico o no tenían formación en TIC. En tercer lugar, se hallaron diferencias significativas en el profesorado que utilizaba las tecnologías digitales en su práctica docente. Por último, también se encontraron diferencias entre el profesorado según la etapa educativa en la que impartía docencia. 


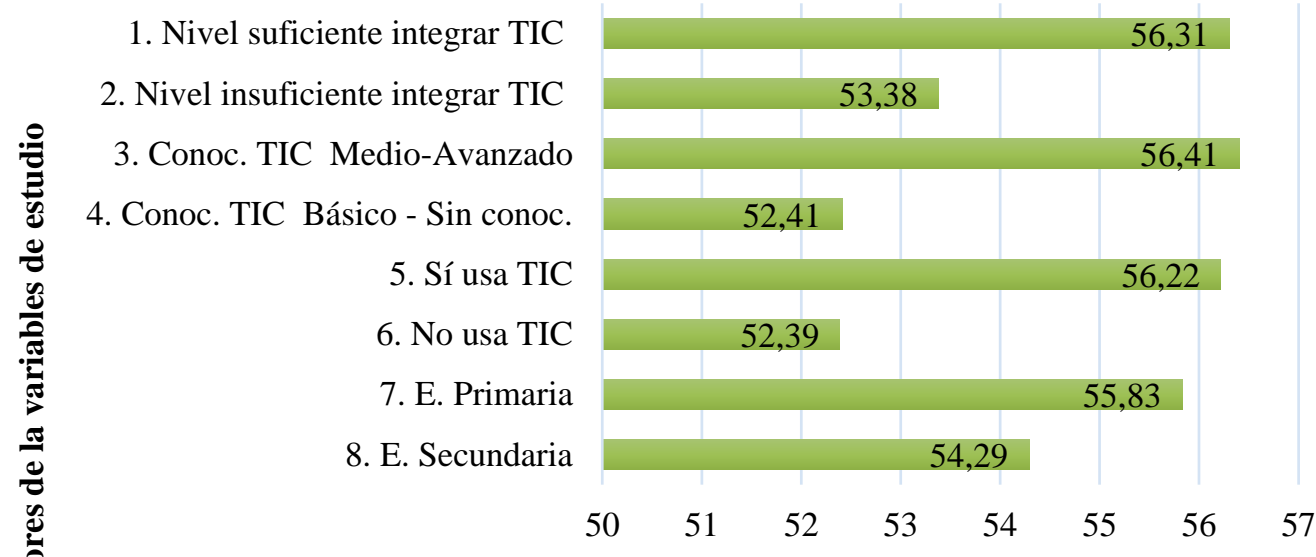

Puntuación total de los ítems de la variable "incidencia postivia de las TIC"

Figura 1. Relación entre las puntuaciones de los 18 ítems de la variable "incidencia positiva de las TIC en el proceso educativo" y las variables "consideración del nivel que posee para integrar las TIC en la práctica docente"

Nota:suficiente: (1), insuficiente (2), "nivel de conocimientos del profesorado en materia TIC": nivel medio o avanzado (3), nivel básico o sin conocimientos en TIC (4), "uso de las TIC en la práctica docente": Sí utiliza las TIC (5), no utiliza las TIC (6), "etapa educativa en la que imparte docencia": primaria (7), secundaria (8). El rango del sumatorio de las puntuaciones de la variable "incidencia positiva de las TIC en el proceso educativo" está entre 18 (puntuación mínima) y 72 (puntuación máxima).

Para tener una visión más amplia de la variable "incidencia positiva de las TIC en los procesos educativos" se calculó la suma de los 18 ítems que la componían. Con ello se pudo obtener una sola puntuación para evaluar y posteriormente analizar la variable en su conjunto.

Como muestra la figura 1, el profesorado que consideraba tener un nivel suficiente para integrar las TIC en la práctica docente valoró en mayor medida que las TIC incidieron positivamente en los procesos educativos $[M=56,31,(D T=8,60)]$ frente al profesorado que consideraba no tener un nivel suficiente $[M=53,38(D T=7,21)]$. Las diferencias fueron significativas [t $(741)=4,83 ; \mathrm{p}=, 00]$.

Los docentes que utilizaron las tecnologías digitales en el aula, a diario, alguna vez a la semana u ocasionalmente, valoró más positivamente la incidencia de las TIC [M= $56,22(D T=8,10)]$ que quienes nunca las utilizaron $[M=52,39(D T=7,84)]$, [t $(741)=$ $5,82 ; \mathrm{p}=, 00]$.

Quienes tenían un nivel avanzado o medio en materia TIC valoraron en mayor medida la incidencia positiva de las tecnologías digitales $[M=56,41(D T=7,84)]$ frente a quienes tenían un nivel básico o no tenían conocimientos en TIC $[M=52,41$ (DT = $8,32)],[\mathrm{t}(742)=6,31 ; \mathrm{p}=, 00]$.

De la misma manera, se observó que el profesorado de educación primaria tuvo una opinión más positiva de la incidencia de las TIC en la práctica educativa $[M=55,83$ $(D T=8,10)]$ que el profesorado de secundaria $[M=54,29(D T=8,30)]$, $[\mathrm{t}(742)=6,31$; $\mathrm{p}=, 00]$.

No se hallaron diferencias significativas entre la valoración de los docentes sobre la incidencia positiva de las TIC y las variables "género del profesorado", "situación administrativa", es decir, personal interino o definitivo en el centro, y "edad del profesorado". En cualquier caso los docentes más jóvenes obtuvieron mayores 
puntuaciones que el resto de edades en la incidencia positiva del uso educativo de las TIC, pero sin llegar a diferir de manera significativa.

\section{Discusión y conclusiones}

El objetivo de este trabajo fue conocer la evolución, entre 2009 y 2016, de cómo el profesorado fue modificando su percepción sobre la incidencia que las TIC tenían en el proceso de enseñanza y aprendizaje. Fruto del análisis de estos resultados se detectaron tres ámbitos bien diferenciados donde las TIC afectaron, a juicio de los docentes, en diferente grado en el proceso educativo. Estos tres ámbitos fueron: la incidencia en el proceso de aprendizaje, la incidencia en el proceso de enseñanza y en la incidencia en la atención a la diversidad del alumnado.

Teniendo como referencia estos tres ámbitos, los resultados de la investigación evidenciaron dos tendencias principales en cuanto a la incidencia positiva de las tecnologías digitales en la práctica educativa: por un lado, el estable valor transmisivo que siguieron aportando las tecnologías a la práctica docente $\mathrm{y}$, por otro lado, la incipiente apreciación del uso de las TIC en los procesos de aprendizaje.

Con relación al primer hallazgo, el profesorado, sin variación en el transcurso de estos años, siguió percibiendo que la incorporación de las tecnologías digitales incidían principalmente en facilitar el acceso a la información, en suscitar el interés y la motivación del alumnado y en promover la participación del alumnado. Estas valoraciones fueron comunes entre el profesorado en 2009 y 2016. Estos datos fueron coincidentes con los resultados encontrados en Cebrián y Ruiz, (2008), Boza et al. (2009) y Pérez, Aguaded y Fandos (2009). Los resultados, muestran una clara visión de los beneficios de las tecnologías digitales tanto en cuanto que son válidos para ayudar al profesor en su práctica docente, como apoyo o complemento a la tarea docente. En esta misma línea, Almerich, Suárez, Orellana y Díaz (2010) afirman que "el profesorado utiliza las TIC más como una forma de apoyo a sus métodos de enseñanza tradicional, que como transformación de su propio método", (p.43). En consecuencia, refleja el continuismo del modelo transmisivo de enseñanza. "Se distingue el valor instructivo que los docentes le conceden a los recursos tecnológicos" Ramírez, Cañedo y Clemente, 2012, p. 152). Estos enfoques transmisivos, por otra parte, "otorgan escasa importancia tanto a los procesos de construcción del aprendiz y a la consideración de lo que el aprendiz aporta a su proceso de aprendizaje, como a los procesos sociales de ayuda y soporte" (Onrubia, 2016, p8).

Con relación al segundo hallazgo, la percepción de los docentes sobre la incidencia positiva de las TIC fue menos valorada en lo relativo a los procesos de aprendizaje, más centrado en la actividad del alumno. No obstante, es remarcable el aumento significativo del número de docentes que aseveraron que las TIC mejoran la adquisición de conocimientos en su alumnado, son indispensables en la práctica educativa, e imprescindibles para desenvolverse en la sociedad. Este resultado es una puerta que se abre a los nuevos modos de entender las TIC como un recurso fundamental en los procesos de aprendizaje. Además, como se observa en esta evolución entre 2009 y 2016, el profesorado fue creando nuevas vías en el uso de las TIC para atender a la diversidad.

Por último, como resultado de esta investigación, se distinguieron cuatro variables que se relacionaron con la opinión del profesorado sobre la incidencia positiva de las TIC en la educación. Estas fueron el uso de las TIC en el aula, la etapa educativa en la que impartieron docencia, el nivel de formación del profesorado en materia TIC, y la consideración de tener un nivel suficiente para integrar las TIC en su práctica docente.

Tal fue así que el profesorado que utilizó las TIC en su práctica docente a diario, 
alguna vez a la semana u ocasionalmente valoró en mayor medida la incidencia positiva de las TIC en la práctica educativa. "Las creencias del profesor sobre el significado de la tecnología en la educación influye en la frecuencia con la que la usa en el aula", (Aguaded, Tirado y Cabero, 2008, p. 196). También se encontró que los docentes con un nivel medio o avanzado en tecnologías digitales mostraron una actitud más positiva hacia la incidencia de éstas en el aula. "La creencia de los profesores en su competencia digital parece explicar también la probabilidad de que utilicen los recursos de la Red en sus prácticas docentes", (Ramírez, Cañedo y Clemente, 2012, p.153). Asimismo, el profesorado que consideraba tener un nivel suficiente para integrar las TIC en su práctica docente hizo una valoración más positiva de la incidencia de éstas en la educación. "El papel de las creencias sobre la atribución de la propia competencia parece ser determinante en la adopción de los recursos" (Ramírez, Cañedo y Clemente, 2012, p.153). Por último, cabe señalar la visión más positiva del profesorado de educación primaria, frente al de secundaria, en la repercusión de las TIC en el proceso de enseñanza y aprendizaje.

Al relacionar la incidencia positiva de las TIC entre el profesorado según el género, la situación administrativa, es decir, personal interino o definitivo en el centro, y la edad del profesorado, no se hallaron diferencias significativas en sus valoraciones.

Para seguir profundizando en este tema sería importante avanzar en el estudio de las variables que se relacionan con el profesorado con actitudes positivas frente a las TIC pero que no las utilizan en su práctica docente.

\section{Referencias}

Aguaded, J. I., Tirado, R., \& Cabero, J. (2008). Los centros TIC en Andalucía, España: un modelo de implicación del profesorado en la integración curricular de la tecnología. Revista Internacional de Ciencias Sociales y Humanidades, SOCIOTAM, 18(2), 171-199.

Aguaded, J. I. \& Pérez, M. A. (2010). Hacia una integración curricular de las TIC en los centros educativos andaluces de primaria y secundaria. Bordón, 62(4), 7-23.

Área, M. (2005). Tecnologías de la información y comunicación en el sistema escolar. Una revisión de las líneas de investigación. Revista Relieve. 11(1), 3-25.

Area, M. (2010). El proceso de integración y uso pedagógico de las TIC en los centros educativos. Un estudio de casos. Revista de educación, 352, 77-97.

Area, M., Borrás, J. F., Castro, F. M., Cepeda, O., Fariña, E., González, D., \& Sanabria, A. L. (2013). Políticas educativas y buenas prácticas TIC: actas II Simposio Internacional SITIC.

Area, M., Sanabria, A. L. \& Vega, A. M. (2015). Las políticas educativas TIC (Escuela 2.0) en las Comunidades Autónomas de España desde la visión del profesorado. Campus virtuales, 2(1), 74-88. https://goo.gl/SZk6S1 (2016-1-9).

Almerich, G., Suárez, J. M., Orellana, N., \& Díaz, M. I. (2010). La relación entre la integración de las tecnologías de la información y comunicación y su conocimiento. Revista de Investigación Educativa, 28(1), 31-50. https://goo.gl/YQbhMH (2013-2-5).

Bisquerra, R. (2009). Metodología de la investigación educativa (2 ${ }^{a}$ edición). Madrid: La Muralla S.A. 
Boza, A., Toscano, M. \& Méndez, J.M. (2009). El impacto de los proyectos TICS en la organización y los procesos de enseñanza-aprendizaje en los centros educativos. Revista de Investigación Educativa. 27(1), 263-289.

Boza, Á., Tirado, R., \& Guzmán, M. D. (2010). Creencias del profesorado sobre el significado de la tecnología en la enseñanza: influencia para su inserción en los centros docentes andaluces. Relieve, 16(1), 1-24.

Boza, A., \& Toscano, M. O. (2011). Buenas prácticas de la integración de las TIC en educación. In VI Congreso virtual de aidipe.

Buendía, L., Colás, M.P. y Hernández, F. (1997). Métodos de investigación en Psicopedagogía. Madrid: McGraw-Hill.

Cabero, J. (2010). La satisfacción de los estudiantes en red en la formación semipresencial/Online Students' Satisfaction with Blended Learning. Comunicar, 18(35), 149-157.

Cabero, J., \& Fernández, J. M. (2014). Una mirada sobre las TIC y la Educación Inclusiva. Comunicación y pedagogía: Nuevas tecnologías y recursos didácticos, (279), 38-42.

Cebrián, M. \& Ruiz, J. (2008). Impacto producido por el Proyecto de Centros TIC en CEIP e IES de Andalucía desde la opinión de los docentes. Pixel-Bit Revista de Medios y Educación. 31, 141-154.

Cebrián, M.; Ruiz, J. \& Sánchez, J (2008). Estudio del impacto del Proyecto TIC desde la opinión de los docentes y estudiantes, en los primeros años de su implantación en los centros públicos de Andalucía. Málaga: Gtea.

Cebrian, M., Ruiz, J., \& Palomo, R. (2011). El impacto de las TIC en los centros educativos. Ejemplo de buenas prácticas. Revista de Docencia Universitaria, 9(1), 245-246.

Chiero, R. T. (1997). Teachers' perspectives on factors that affect computer use. Journal of Research on Computing in Education, 30(2), 133-145.

Colás, P., De Pablos, J., González, A., \& Conde, J. (2009). Impacto de las Políticas TIC en la Integración Curricular de los Recursos Tecnológicos. In XIV Congreso Nacional de Modelos de Investigación Educativa. 14, 1133-1139.

De Pablos, J., Colás, P., \& Villaciervos, P. (2010). Políticas educativas y buenas prácticas con TIC en la comunidad autónoma andaluza. Teoría de la Educación. Educación y Cultura en la Sociedad de la Información, 11(1), 180-202. https://goo.g1/JrGZIF

Fu, J. S. (2013). ICT in education: A critical literature review and its implications. International Journal of Education and Development using Information and Communication Technology, 9(1), 112.

Hermans, R., Tondeur, J., van Braak, J., \& Valcke, M. (2008). The impact of primary school teachers' educational beliefs on the classroom use of computers. Computers \& Education, 51(4), 1499-1509.

Jimoyiannis, A., \& Komis, V. (2007). Examining teachers' beliefs about ICT in education: Implications of a teacher preparation programme. Teacher development, 11(2), 149-173. 
Onrubia, J. (2016). Aprender y enseñar en entornos virtuales: actividad conjunta, ayuda pedagógica y construcción del conocimiento. Revista de Educación a Distancia, (50).

Paraskeva, F., Bouta, H., \& Papagianni, A. (2008). Individual characteristics and computer self-efficacy in secondary education teachers to integrate technology in educational practice. Computers \& Education, 50(3), 1084-1091.

Pérez, M. A., Aguaded, J. I., \& Fandos, M. (2009). Una política acertada y la formación permanente del profesorado, claves en el impulso de los centros TIC de Andalucía (España). Estudios pedagógicos (Valdivia), 35(2), 137-154.

Ramírez, E., Cañedo, I., \& Clemente, M. (2012). Las actitudes y creencias de los profesores de secundaria sobre el uso de Internet en sus clases. Comunicar: Revista Científica de Comunicación y Educación, 19(38), 147-155.

Ruiz, J. \& Ortega, J.M. (2009). Integration of ICT in Primary and Secondary Schools in Andalusia, Spain. pp. 133-137. En: Proceedings of the IASK International Conference: Teaching and Learning 2009: 7-9 diciembre 2009, Porto, Portugal. Porto: International Association for the Scientific Knowledge.

Sang, G., Valcke, M., van Braak, J., \& Tondeur, J. (2010). Student teachers' thinking processes and ICT integration: Predictors of prospective teaching behaviors with educational technology. Computers \& Education, 54(1), 103-112.

Tirado R. \& Aguaded, I. (2014) Influencias de las creencias del profesorado sobre el uso de la tecnología en el aula. Revista de Educación, 363. https://goo.gl/1SHtJa

Valverde, J., \& Sosa, M. J. (2014). Centros educativos e-competentes en el modelo 1: 1: el papel del equipo directivo, la coordinación TIC y el clima organizativo.

Viherä, M.L., \& Nurmela, J.. (2001). Communication capability as an intrinsic determinant for information age. Futures, 33, 245-265.

Windschitl, M. \& Sahl, K. (2002). “Tracing Teachers' Use of Technology in a Laptop Computer School: The Interplay of Teacher Beliefs, Social Dynamics, and Institutional Culture". American Educational Research Journal, Spring 2002, Vol. 39, No. 1, pp. 165-205

Fecha de recepción: 05/05/2017

Fecha de revisión: 01/06/2017

Fecha de aceptación: 28/06/2017 
López-Gonzales, J.B., Ortega-Tuleda, J.M. 